ThEOREM 4.2. A complete regular ring $S$ is continuous if and only if $\bar{S}_{l}=\bar{S}_{r}$.

By [3, Theorem 3] every continuous regular ring is (both left and right) self injective if it contains no nonzero strongly regular ideals. Hence we have

THEOREM 4.3. Let $S$ be a complete regular ring, and suppose that $S$ does not contain any nonzero strongly regular ideals. Then $\bar{S}_{l}=\bar{S}_{r}$ (if and) only if $S$ is self injective, that is, $S=\bar{S}_{l}=\bar{S}_{r}$.

\title{
REFERENCES
}

1. R. E. Johnson, The extended centralizer of a ring over a modvle, Proc. Amer. Math. Soc. 2 (1951), 891-895.

2. Y. Utumi, On quotient rings, Osaka Math. J. 8 (1956), 1-18.

3. - On continuous regular rings and semisimple self injective rings, Canad. J. Math. 12 (1960), 597-605.

4. - On continuous regular rings, Canad. Math. Bull. 4 (1961), 63-69.

UNIVERSITY OF ROCHESTER

\section{INVERSE LIMITS OF SOLVABLE GROUPS}

ETHAN D. BOLKER

In this paper we generalize to groups of Galois type some results of $\mathrm{P}$. Hall on finite solvable groups $[1 ; 2 ; 3]$. We need, in a modified form, some results of van Dantzig: the definition of supernatural numbers (which are related to van Dantzig's universal numbers) and Theorem 5, which he proved for ordinary p-Sylow subgroups [6]. Lemmas 1 and 4 and the method of proof in Theorem 5 are due to Tate [5].

A topological group $G$ is of Galois type if it is compact and totally disconnected. In any Galois type group the open normal subgroups form a neighborhood base at the identity. Every closed subgroup is the intersection of the open subgroups containing it [4]. Whenever $M$ and $N$ are open normal subgroups of $G$ and $N \supset M$ we shall write $\phi_{N}^{M}$ for the natural homomorphism of $G / M$ onto $G / N$ (these quotient groups are finite) and $\phi_{N}$ for the natural homomorphism of $G$ onto $G / N . G$ is the inverse limit of the groups $\{G / N\}, N$ ranging over the open normal subgroups of $G$. Conversely, the inverse limit of finite groups is of Galois type. 1962.

Presented to the Society, August 30,1962; received by the editors January 31, 
A supernatural number is a formal product of powers of primes. Infinitely many factors are permitted, with exponents $1,2, \cdots, \infty$. Supernatural numbers are multiplied by addition of exponents. If $\pi$ is a set of primes and $\delta$ a supernatural number we say

$$
\begin{array}{ll}
\delta \text { and } \pi \text { are coprime } & \text { when } \\
\pi \mid \delta(\pi \text { divides } \delta) & \text { when } p \in \pi \text { implies } p \nmid \delta, \\
\delta \mid \pi & \text { when } p \mid \delta \text { implies } p \in \pi .
\end{array}
$$

Whenever $G$ is a group of Galois type and $S$ is a closed subgroup of $G$ we define the index of $S$ in $G([G: S])$ as the supernatural number which is the least common multiple of the integers $[G: U]$ as $U$ ranges over open subgroups of $G$ containing $S$. Then $[G: S]$ is also the l.c.m. $\{[G: S N]\}$ as $N$ ranges over the open normal subgroups of $G$. The order of $G, o(G)$, is $[G: 1]$. $G$ is a $\pi$ group if $o(G) \mid \pi$. A closed subgroup $S$ of $G$ is a $\pi$-Sylow subgroup if $\pi$ and $[G: S]$ are coprime and $S$ is a $\pi$ group.

1. Lемма. If $S$ and $T$ are closed subgroups of the Galois type group $G$ and $S$ contains $T$ then $[G: T]=[G: S][S: T]$.

Proof. For $N$ an open normal subgroup of $G$ we know [G:TN] $=[G: S N][S N: T N]$ and $[S N: T N]=[S: T(S \cap N)]$. Taking the least common multiples as $N$ varies we see that $[G: T]$ divides $[G: S][S: T]$, so we need only show that $[G: S][S: T]$ divides $[G: T]$. Suppose that for $m$ and $k<\infty, p^{m}$ divides $[G: S]$ and $p^{k}$ divides $[S: T]$. It follows that there are open normal subgroups $N_{1}$ and $N_{2}$ of $G$ for which $p^{m} \mid\left[G: S N_{1}\right]$ and $p^{k} \mid\left[S: T\left(S \cap N_{2}\right)\right]$. Let $N=N_{1} \cap N_{2}$. Then $p^{m} \mid[G: S N]$ and $p^{k} \mid[S: T(S \cap N)]$. Therefore $p^{m+k}$ divides $[G: T N]$ and thus also divides $[G: T]$. Therefore $[G: S][S: T]$ divides $[G: T]$. q.e.d.

2. Corollary. A subgroup $S$ is a $\pi$-Sylow subgroup for some $\pi$ if and only if its order and index are coprime.

3. Corollary. One $\pi$-Sylow subgroup cannot strictly contain another.

Proof. Let $S$ and $T$ be $\pi$-Sylow subgroups for which $T \subset S$. Then $o(S)=o(T)[S: T]$ implies $[S: T]$ divides $\pi$, but $[G: T]=[G: S][S: T]$ implies $[S: T]$ and $\pi$ are coprime, which is a contradiction unless $S=T$.

4. LEMMA. If $S$ is the intersection of a decreasing family $\left\{S_{\alpha}\right\}$ of closed subgroups of $G$ then $[G: S]=$ l.c.m. $\left\{\left[G: S_{\alpha}\right]\right\}$.

Proof. Lemma 1 implies $[G: S]=\left[G: S_{\alpha}\right]\left[S_{\alpha}: S\right]$ for all $\alpha$, hence 
1.c.m. $\left\{\left[G: S_{\alpha}\right]\right\}$ divides [G:S]. Conversely, if $m<\infty$ and $p^{m} \mid[G: S]$ then there is an open normal subgroup $N$ for which $p^{m} \mid[G: S N]$. Since $G-S N$ is compact and disjoint from $S=\cap S_{\alpha}$ there is a finite intersection $S_{\alpha_{1}} \cap \cdots \cap S_{\alpha_{k}}$ contained in $S N$. However $S_{\alpha_{1}} \cap \cdots \cap S_{\alpha_{k}}$ equals $S_{\alpha_{k}}$ since the $S_{\alpha}$ are decreasing. Therefore $p^{m} \mid\left[G: S_{\alpha_{k}}\right]$, hence $p^{m} \mid$ 1.c.m. $\left\{\left[G: S_{\alpha}\right]\right\}$. Thus $[G: S]=1$. .m. $\left\{\left[G: S_{\alpha}\right]\right\}$. q.e.d.

A group of Galois type is projectively solvable if it is the inverse limit of finite solvable groups.

5. ThEOREM. Let $G$ be projectively solvable. For each $\pi$ dividing $o(G)$, $G$ has $\pi$-Sylow subgroups, any two are conjugate, and any $\pi$ subgroup of $G$ is contained in one of them.

Proof. Let $\mathfrak{T}$ be the set of closed subgroups $S$ of $G$ for which $[G: S]$ and $\pi$ are coprime. $G \in \mathscr{N}$ and $\mathscr{N}$ is partially ordered by inclusion. Lemma 4 shows that the intersection $S$ of a maximal chain from $\mathfrak{T}$ is minimal in $\mathscr{T}$. We shall show such an $S$ is necessarily a $\pi$ group.

Let $N$ be an open normal subgroup of $G$. We must show $o(S / S \cap N)$ divides $\pi$. Since $G / N$ is solvable so is $S / S \cap N$, which is isomorphic to $S N / N$. Choose a $\pi$-Sylow subgroup $W$ of $S / S \cap N$ (Hall, [1]). Let $T$ be the inverse image in $S$ of $W$ under $\phi_{S \cap N}$. T is closed in $G$ and both open and closed in $S$. [G:T] $=[G: S][S: T]$. [G:S] and $\pi$ are coprime since $S \in \mathscr{N}$. Since $\pi$ and $[S / S \cap N: W]$, which equals $[S: T]$, are coprime, we see that $[G: T]$ and $\pi$ are coprime. Therefore $T \in \mathscr{N}$. Since $T \subset S$ and $S$ is minimal in $\Re, T=S$. Therefore $S / S \cap N$ $=T / S \cap N=W$, but we know $o(W)$ divides $\pi$. Therefore $S$ is a $\pi$ Sylow subgroup of $G$.

Now let $T$ be any $\pi$ subgroup of $G$. For each open normal subgroup $N$ of $G$ the set $K_{N}$ of members of $G$ whose images $\sigma$ under $\phi_{N}$ satisfy $\sigma^{-1} \phi_{N}(T) \sigma \subset \phi_{N}(S)$ is closed and nonempty (Hall, [1]). $N \subset M$ implies $K_{N} \subset K_{M}$, hence $\left\{K_{N}\right\}$ has the finite intersection property. If $\tau \in \cap K_{N}$ then $\tau^{-1} T \tau \subset S$. Therefore any $\pi$ group has a conjugate inside a given $\pi$-Sylow subgroup. Corollary 3 then implies any two $\pi$-Sylow subgroups are conjugate. q.e.d.

6. CoRollary. Let $\Re(S)$ be the normalizer of the $\pi$-Sylow subgroup $S$. Then $[G: \Re(S)]$ and $\pi$ are coprime since $S \subset \Re(S)$.

Theorem 5 guarantees the existence of $\pi$-Sylow subgroups. Zorn's lemma was required in the proof. Once this existence has been established, however, the $\pi$-Sylow subgroups can be characterized somewhat more constructively.

7. THEOREM. Each $\pi$-Sylow subgroup $S$ of $G$ may be written as the 
inverse limit of groups $\left\{S_{N}\right\}$ where $S_{N}$ is a $\pi$-Sylow subgroup of $G / N$ and $\phi_{N}^{M}$ maps $S_{M}$ onto $S_{N}$. Conversely, if $\left\{S_{N}\right\}$ is a family of subgroups of the $G / N$ with these properties then the inverse limit $S$ of $\left\{S_{N}\right\}$ is a $\pi$-Sylow subgroup of $G$.

Proof. Any closed subgroup $S$ of $G$ may be written as the inverse limit of $\left\{S_{N}\right\}$ where $S_{N}=S N / N$, which is isomorphic to $S / S \cap N$. Then $\phi_{N}^{M}$ maps $S_{M}$ onto $S_{N}$. The argument in Theorem 5 shows that $S_{N}$ is a $\pi$-Sylow subgroup of $G / N$ if $S$ is a $\pi$-Sylow subgroup of $G$.

Conversely, if each $S_{N}$ is a $\pi$-Sylow subgroup then $S$ is a $\pi$ group, hence $S$ is contained in some $\pi$-Sylow subgroup $S^{\prime}$. Then $S^{\prime} N / N$ must equal $S N / N$ for all $N$, hence $S=S^{\prime}$. q.e.d.

We can now generalize Hall's results on Sylow systems [3]. Let $G$ be projectively solvable, $\pi_{G}$ the set of primes dividing $o(G)$. Choose a $\pi_{G}-\{p\}$-Sylow subgroup ( $p$-complement) $C_{p}$ for each $p \in \pi_{G}$. For $\Sigma \subset \pi_{G}$ let $S_{\Sigma}=\bigcap_{p \notin \Sigma} C_{p}$. We set $S_{\pi_{G}}=G$. Then $\mathcal{S}=\left\{S_{\Sigma}\right\}$ is a Sylow system of $G$.

\section{TheOREM. $S_{\Sigma}$ is a $\Sigma$-Sylow subgroup of $G$.}

Proof. Each $C_{p}$ is the inverse limit of $p$-complements of the groups $G / N$ (Theorem 7). Hall's theorem [3] tells us that the intersections of the $p$-complements for $p \notin \Sigma$ are $\Sigma$-Sylow subgroups of the groups $G / N$. These $\Sigma$-Sylow subgroups are mapped onto each other by the $\phi_{N}^{M}$, hence their inverse limit is a $\Sigma$-Sylow subgroup which is easily proved equal to $S_{\Sigma}$. q.e.d.

9. LemMa. If $\left\{\Sigma_{\alpha}\right\}$ is a family of subsets of $\pi_{G}$ then $S_{\cap \Sigma_{\alpha}}=\cap S_{\Sigma_{\alpha}}$.

This follows trivially from the definition of $S_{\Sigma}$.

10. THEOREM. $S_{\Sigma} S_{\Delta}=S_{\Sigma \cup \Delta}$.

Proof. If $\sigma \delta \in S_{\Sigma} S_{\Delta}$ each of its projections $\phi_{N}(\sigma \delta)$ lies in the product of a $\Sigma$-Sylow subgroup and a $\Delta$-Sylow subgroup of $G / N$ (Theorem 7). By Hall's theorem [3] this product is a $\Sigma \cup \Delta$-Sylow subgroup of $G / N$, and is in fact $\phi_{N}\left(S_{\Sigma \cup \Delta}\right)$. Hence $S_{\Sigma} S_{\Delta} \subset S_{\Sigma \cup \Delta}$.

To prove the reverse inclusion, suppose $\tau \in S_{\Sigma U_{\Delta}}$. Then for each open normal subgroup $N, \phi_{N}(\tau) \in \phi_{N}\left(S_{\Sigma U_{\Delta}}\right)$, which equals $\phi_{N}\left(S_{\Sigma}\right) \phi_{N}\left(S_{\Delta}\right)$. Thus there are elements $\sigma_{N} \in S_{\Sigma}$ and $\delta_{N} \in S_{\Delta}$ for which $\phi_{N}\left(\sigma_{N} \delta_{N}\right)=\phi_{N}(\tau)$. This shows that the set $K_{N}$ of those members $\eta$ of $S_{\Sigma} S_{\Delta}$ for which $\phi_{N}(\eta)=\phi_{N}(\tau)$ is nonempty. $K_{N}$ is closed since $S_{\Sigma} S_{\Delta}$ is closed, even though we do not yet know it is a subgroup. $\left\{K_{N}\right\}$ has the finite intersection property since for open normal subgroups $N_{1}, \cdots, N_{j}, \cap K_{N_{i}} \supset K_{\cap N_{\imath}}$. Choose $\rho \in \cap K_{N}$. For all $N, \phi_{N}(\rho)=\phi_{N}(\tau)$ implies $\rho=\tau$, but we know $\rho \in S_{\Sigma} S_{\Delta}$. Therefore $S_{\Sigma \cup \Delta} \subset S_{\Sigma} S_{\Delta}$. q.e.d. 
11. Corollary. $S_{\Sigma} S_{\Delta}=S_{\Sigma U_{\Delta}}=S_{\Delta} S_{\Sigma}$, i.e., $\delta$ consists of commuting subgroups. In particular, $\left\{S_{\{p\}}\right\}$ is a commuting family of ordinary p-Sylow subgroups.

12. Corollary. Theorem 10 is true for infinite products, i.e.,. $\left[S_{\Sigma_{\alpha}} S_{\Sigma_{\beta}} \cdots\right]-=S \cup \Sigma_{\alpha}$ for any family $\left\{\Sigma_{\alpha}\right\}$ of subsets of $\pi_{G}$.

Proof. By $S_{\Sigma_{\alpha}} S_{\Sigma_{\beta}}$. . we mean the set of products of finitely many members of $U S_{\Sigma_{\alpha}}$. Since the $S_{\Sigma_{\alpha}}$ commute pairwise, this set, and hence its closure, $S$, is a subgroup. $S$ is the smallest closed subgroup containing $S_{\Sigma_{\alpha}}^{\prime}$ for all $\alpha$. Since $S \cup \Sigma_{\alpha} \supset S_{\Sigma_{\alpha}}$ for all $\alpha$ we have $S \cup \Sigma_{\alpha} \supset S$. It will suffice to prove that $S$ is a $U \Sigma_{\alpha}$-Sylow subgroup, for then we may apply Theorem 8 and Corollary $3 . S$ is a $U \Sigma_{a}$ group since it is contained in one. Since $\left[G: S_{\Sigma_{\alpha}}\right]$ and $\Sigma_{\alpha}$ are coprime and $\left[G: S_{\Sigma_{\alpha}}\right]$ $=[G: S]\left[S: S_{\Sigma_{\alpha}}\right]$ we see that for each $\alpha,[G: S]$ and $\Sigma_{\alpha}$ are coprime. Thus $[G: S]$ and $U \Sigma_{\alpha}$ are coprime and $S$ is a $U \Sigma_{\alpha}$-Sylow subgroup. q.e.d.

By combining the results 8 through 12 we prove

13. THEOREM. For each projectively solvable group $G$ there is a lattice injection of the set of subsets of $\pi_{G}$ into the lattice of closed subgroups of $G$. Its range is commutative and consists of one Sylow subgroup of each type. The lattice join corresponds to set multiplication.

We now study the family of Sylow systems. The Sylow system $\tau^{-1} \delta \tau$ generated by the $\tau$-conjugates of the $p$-complements in $S$ is said to be conjugate to $S$. If $\delta^{*}=\tau^{-1} \delta \tau$ then $S_{\Sigma}^{*}=\tau^{-1} S_{\Sigma} \tau$ for all $\Sigma \subset \pi_{G}$.

14. Conjugacy theorem. Any two Sylow systems $s$, $\mathcal{S}^{*}$ are conjugate.

Proof. For any finite set $\left\{p_{1}, \cdots, p_{n}\right\} \subset \pi_{G}$ there is a $\tau \in G$ for which $\tau^{-1} C_{p_{i}} \tau=C_{p_{i}}^{*}, i \leqq n$. We prove this by induction. The proof is the same as Hall's [3]. For $n=1$ the statement is Theorem 5. Assume that conjugation with $\tau$ takes $C_{p_{i}}$ into $C_{p_{i}}^{*}, i \leqq n-1$. Since $S_{p_{n}}^{*} C_{p_{n}}^{*}$ $=G=C_{p_{n}}^{*} S_{p_{n}}^{*}$ any conjugate of $C_{p_{n}}^{*}$ may be obtained by using an element of $S_{p_{n}}^{*}$. In particular, there is a $\sigma \in S_{p_{n}}^{*}$ for which $\sigma^{-1}\left(\tau^{-1} C_{p_{n}} \tau\right) \sigma$ $=C_{p_{n}}^{*}$. Since $S_{p_{n}}^{*} \subset C_{p}^{*}$ when $p \neq p_{n}$ we can conclude that $(\tau \sigma)^{-1} C_{p_{i}}(\tau \sigma)$ $=C_{p_{i}}^{*}$ for $i \leqq n$.

Let $K_{p}$ be the coset of the normalizer of $C_{p}$ which takes $C_{p}$ to $C_{p}^{*}$. $K_{p}$ is closed. The induction above shows $\left\{K_{p}\right\}$ has the finite intersection property. Any $\tau \in \cap K_{p}$ clearly satisfies our requirements. q.e.d.

15. EMBEDDING THEOREM. If $\Im$ is a commuting set of subgroups of

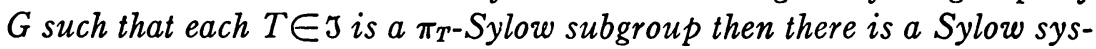
tem $\mathrm{S}$ containing $\Im$. 
Proof. For each $p \in \pi_{G}$ let $T_{p}$ be the closure of the product of those $T \in J$ for which $p \nmid o(T)$. (If there are no such $T$ let $T_{p}=\{e\}$.) The argument in Corollary 12 shows that $T_{p}$ is a group. $T_{p}$ is the inverse limit of the product in each $G / N$ of the groups $\phi_{N}(T)$ for which $p \nmid o(T)$, therefore $p \nmid o\left(T_{p}\right)$. Using Theorem 5 , choose a $p$-complement $C_{p}$ containing $T_{p}$.

Let $\mathcal{S}$ be the Sylow system generated by $\left\{C_{p}\right\}$. For each $T \in J$, $S_{\pi_{T}}$ contains $T$ since $S_{\pi_{T}}$ is the intersection of the $C_{p}$ for which $p \notin \pi_{T}$, i.e., for which $T \subset T_{p} \subset C_{p}$. Since both $T$ and $S_{\pi_{T}}$ are $\pi_{T-}$ Sylow subgroups they are equal, hence $J \subset s$. q.e.d.

16. SubGroup theOREM. If $H$ is a closed subgroup of $G$ and $J$ is a Sylow system for $H$ then there is a Sylow system $S$ of $G$ for which $\mathfrak{J}=\mathrm{S} \cap H$, i.e., each $T \in \mathcal{J}$ is $S \cap H$ for some $S \in \mathcal{S}$.

Proof. Let $\left\{C_{p}^{\prime}\right\}$ be the $p$-complements in $J$. For each $p \in \pi_{H}$ choose a $p$-complement $C_{p}$ (in $G$ ) which contains $C_{p}^{\prime}$. If $p \notin \pi_{H}$ choose a $p$-complement $C_{p}$ containing $H$. Then the Sylow system generated by $\left\{C_{p}\right\}$ will do. Suppose $T \in J$. Then $S_{x_{T}} \cap H$ contains $T$ and is a $\pi_{T}$ group. $[H: T]=\left[H: S_{\pi_{T}} \cap H\right]\left[S_{\pi_{T}} \cap H: T\right]$ and $T$ a $\pi_{T_{T}}$-Sylow subgroup imply $\left[H: S_{\boldsymbol{x}_{T}} \cap H\right]$ and $\pi_{T}$ are coprime. Thus $S_{\boldsymbol{x}_{\boldsymbol{T}}} \cap H$ is a $\pi_{T}$-Sylow subgroup and therefore equals $T$. q.e.d.

\section{REFERENCES}

1. P. Hall, $A$ note on soluble groups, J. London Math. Soc. 3 (1928), 98.

2. - A characteristic property of soluble groups, J. London Math. Soc. 12 (1937), 198.

3. - On the Sylow systems of a soluble group, Proc. London Math. Soc. 43 (1937), 316.

4. L. Pontrjagin, Topological groups, Chapter III, pp. 77-80, Princeton Univ. Press, Princeton, N. J., 1939.

5. Séminaire Bourbaki $1959 / 60$, No. 189 , Cohomologie des groupes compacts totalement discontinus: A. Douady, from notes by S. Lang on an unpublished article by J. Tate.

6. D. van Dantzig, Zur topologischen Algebra. III, Compositio Math. 3 (1936), 408-426.

HARVARD UNIVERSITY 